\title{
Periodontal disease and adverse pregnancy outcomes
}

\author{
Is periodontal disease associated with adverse pregnancy outcomes?
}

\author{
Agueda A, Ramón JM, Manau C, Guerrero A, Echeverría JJ. \\ Periodontal disease as a risk factor for adverse pregnancy \\ outcomes: a prospective cohort study. J Clin Periodontol 2008; \\ 35:16-22
}

Design This was a prospective cohort study.

Cohort selection The initial sample comprised 1404 pregnant women seeking prenatal care at the University Hospital of Lleida (Spain) between March 2003 and January 2005.

Data collection and analysis Periodontal data [full-mouth data, pocket probing depths (PPD) and clinical attachment levels (CAL) and bleeding on probing], pregnancy outcome variables and information on other factors that may influence adverse pregnancy outcomes were collected. Data were analysed using a logistic regression model.

Results The incidence of preterm birth (PB) and low birthweight (LBW) was $6.6 \%$ and $6.0 \%$, respectively. The incidence of preterm LBW (PLBW) was $3.3 \%$. PB was related to mother's age, systemic disease, onset of prenatal care, previous $\mathrm{PB}$, complications of pregnancy, type of delivery, the presence of untreated caries and the presence of periodontitis (odds ratio, 1.77; 95\% confidence interval, 1.08-2.88). LBW was related to the mother's smoking habits, ethnicity, systemic diseases, previous LBW babies, complications of pregnancy and type of delivery. PLBW was related to the mother's age, onset of prenatal care, systemic diseases, previous LBW babies, complications of pregnancy and type of delivery.

Conclusions The factors involved in many cases of adverse pregnancy outcomes have still not being identified, although systemic infections may play a role. This study found a modest association between periodontitis and PB. Further research is required to establish whether periodontitis is a risk factor for PB and/ or LBW.

\section{Commentary}

The authors investigated whether periodontitis was associated with PB, LBW or PLBW in a cohort of 1296 women. They found that periodontitis, defined as the presence of $\geq 4$ teeth with at least one site with $\mathrm{PPD} \geq 4 \mathrm{~mm}$ and $\mathrm{CAL} \geq 3 \mathrm{~mm}$ at the same site, was modestly associated with preterm birth (OR, 1.77). Although several attempts were made to reduce bias and chance, confounding may remain a problem for some strong determinants.

Address for correspondence: Professor Jose J Echeverría, Dental School, University of Barcelona, Feixa Llarga s/n, 08907 L'Hospitalet de Llobregat Barcelona, Spain. E-mail: jjperio@yahoo.es
Smoking exposure was categorised as "never smoker or former smoker before pregnancy", "former smoker of $<15$ cigarettes/ day just until pregnancy", "smoker of $\geq 15$ cigarettes just until pregnancy", "smoker of $<6$ cigarettes during pregnancy" and "smoker of $\geq 6$ cigarettes during pregnancy". This strategy seems comprehensive but it has some limitations. Combining "never smokers" and ex-smokers in a single group is not straightforward because ex-smokers may well include women who quit smoking because they were planning to become pregnant. The effect of this exposure cannot be ruled out by stating that they were not smoking before pregnancy. This is particularly influential because this was the reference group for the analyses. A more accurate assessment of the cumulative exposure to smoking is also missing.

Social dimensions, which have been consistently associated with both poor pregnancy outcomes and periodontitis, were excluded from the multiple logistic regression models. Despite the lack of significant bivariate association in preliminary analyses, however, socioeconomic risk factors ought to be included in the models because they are critical elements in the conceptual model underlying periodontitis and poor pregnancy outcomes. ${ }^{1,2}$

The authors assessed socioeconomic status using employment and it seems that this tool did not capture social variation as reported income could have done, which is the most broadly used social indicator today.

This information must be assessed in the light of associations between periodontitis and poor pregnancy outcomes being considerably confounded by shared causes such as tobacco smoking, the residual confounding effect of which has been previously reported, ${ }^{3}$ and social inequalities - the multidimensional nature of the latter and its many levels is also likely to continue to cause residual confounding after adjustment by one or two variables.

\section{Practice point}

The authors found a modest positive association between periodontitis and preterm birth, but no association with the outcomes low birthweight and preterm low birthweight. The possibility of a residual confounding effect of contextual factors can not be eliminated.

\section{Rodrigo López}

Department of Periodontology, Faculty of Health Sciences, University of Aarhus, Aarhus, Denmark

1. Hosmer DW Lemeshow S. Applied Logistic Regression. 2nd Edn. New York: John Wiley; 2000.

2. von Elm E, Altman DG, Egger M, Pocock SJ, Gøtzsche PC, Vanderbroucke JP. The Strengthening the Reporting of Observational Studies in Epidemiology (STROBE) statement: guidelines for reporting observational studies. Prevent Med 2007; 45:247-251

3. Hujoel PP, Drangsholt M, Spiekerman C, DeRouen TA. Periodontitis-systemic disease associations in the presence of smoking - causal or coincidental? Periodontology $20002002 ; 30: 51-60$

Evidence-Based Dentistry (2008) 9, 48. doi:10.1038/sj.ebd.6400581 